\title{
A multicenter survey on endoscopic retrograde cholangiopan- creatography during the COVID-19 pandemic in northern and central Italy
}

\section{다 (우우}

\section{Authors}

Giulio Donato ${ }^{1}$, Edoardo Forti², Massimiliano Mutignani², Maria Antonella Laterra ${ }^{3}$, Daniele Arese ${ }^{3}$, Franco Coppola ${ }^{3}$, Piera Zaccari ${ }^{4}$, Alberto Mariani ${ }^{4}$, Paolo Giorgio Arcidiacono ${ }^{4}$, Flavia Pigò ${ }^{5}$, Rita Conigliaro ${ }^{5,6}$, Deborah Costa ${ }^{7}$, Alberto Tringali ${ }^{7}$, Alessandro Lavagna ${ }^{8}$, Rodolfo Rocca ${ }^{8}$, Roberto Gabbiadini ${ }^{9}$, Alessandro Fugazza ${ }^{9}$, Alessandro Repici ${ }^{9}$, Giammarco Fava ${ }^{10}$, Francesco Marini ${ }^{10}$, Piergiorgio Mosca ${ }^{10}$, Flavia Urban ${ }^{11}$, Fabio Monica ${ }^{11}$, Stefano Francesco Crinò $^{12}$, Armando Gabbrielli ${ }^{12}$, Matteo Blois ${ }^{13}$, Cecilia Binda ${ }^{14}$, Monica Sbrancia ${ }^{14}$, Carlo Fabbri ${ }^{14}$, Roberto Frego ${ }^{15}$, Marco Dinelli ${ }^{15}$, Venerina Imbesi ${ }^{16}$, Pietro Gambitta ${ }^{16}$, Marco Balzarini ${ }^{17}$, Sergio Segato ${ }^{17}$, Leonardo Minelli Grazioli ${ }^{18}$, Cristiano Spada ${ }^{18}$, Arnaldo Amato ${ }^{19}$, Giovanna Venezia ${ }^{20}$, Giovanni Aragona ${ }^{21}$, Cesare Rosa ${ }^{22}$, Costanza Alvisi' ${ }^{22}$, Massimo Devani ${ }^{23}$, Gianpiero Manes ${ }^{23}$, Iginio Dell'Amico ${ }^{24}$, Carlo Gemme ${ }^{25}$, Raffaella Reati ${ }^{26}$, Francesco Auriemma ${ }^{27}$, Benedetto Mangiavillano ${ }^{27}$, Marcello Rodi ${ }^{28}$, Helga Bertani ${ }^{6}$, Dario Mazzucco ${ }^{29}$, Elia Armellini ${ }^{30}$, Paolo Cantù ${ }^{31}$, Roberto Penagini ${ }^{31}$, Pietro Occhipinti ${ }^{1}$

Institutions

1 Gastroenterology Unit, Department of Oncological and Specialty Medicine, Azienda Ospedaliero-Universitaria Maggiore della Carità, Novara, Italy

2 Digestive and Operative Endoscopy Unit, ASST Grande Ospedale Metropolitano Niguarda, Milan, Italy

3 Gastroenterology Unit, Ospedale San Giovanni Bosco, Torino, Italy

4 Pancreatobiliary Endoscopy and EUS Division, Pancreas Translational and Clinical Research Center, San Raffaele Scientific Institute, Vita Salute San Raffaele University, Milan, Italy

5 Gastroenterology and Digestive Endoscopy - Azienda Ospedaliero-Universitaria Modena, Ospedale di Baggiovara, Italy

6 Digestive Endoscopy Unit, Azienda OspedalieroUniversitaria Modena, Policlinico di Modena, Italy

7 Gastroenterology Unit, ULSS 2 Marca Trevigiana, Ospedale Conegliano-Vittorio Veneto, Italy

8 Gastroenterology Unit, AO Ordine Mauriziano, Torino, Italy

9 Digestive Endoscopy Unit, Humanitas Clinical and Research Center - IRCCS, Rozzano, Milan, Italy

10 SOD Malattie Apparato Digerente, Endoscopia Digestiva, Malattie Infiammatorie Croniche Intestinali, Ospedali Riuniti, Ancona, Italy

11 Gastroenterology Unit, ASU GI Ospedale di Cattinara Trieste, Italy

12 Gastroenterology and Digestive Endoscopy Unit, The Pancreas Institute, Verona, Italy

13 Operative Endoscopy Unit, Azienda UsI Toscana NordOvest, Livorno, Italy
14 Gastroenterology and Digestive Endoscopy Unit, ForlìCesena Hospitals, AUSL Romagna, Italy

15 Operative Endoscopy Unit, ASST Monza, Italy

16 Gastroenterology and Digestive Endoscopy Unit, ASST Ovest Milanese, Legnano, Milan, Italy

17 Gastroenterology Unit, ASST Sette-Laghi, Varese, Italy

18 Digestive Endoscopy Unit, Fondazione Poliambulanza Istituto Ospedaliero, Brescia, Italy

19 Gastroenterology Unit, Ospedale Valduce, Como, Italy

20 Gastroenterology Unit, Azienda Ospedaliera S.Croce e Carle, Cuneo, Italy

21 Gastroenterology and Hepatology Unit, Ospedale Civile, AUSL Piacenza, Italy

22 Digestive Endoscopy Unit, ASST Pavia, Ospedali Voghera-Vigevano, Pavia, Italy

23 ASST Rhodense, Presidio di Rho, Milan, Italy

24 Endoscopy Unit, Azienda UsI Toscana Nord-Ovest, Massa Carrara, Italy

25 Gastroenterology Unit, Azienda Ospedaliera SS. Antonio e Biagio e Cesare Arrigo, Alessandria, Italy

26 ASST Rhodense, Presidio di Garbagnate Milanese, Milan, Italy

27 Gastrointestinal Endoscopy Unit, Humanitas - Mater Domini, Castellanza, Varese, Italy

28 Gastroenterology and Digestive Endoscopy Unit, Ospedale S. Andrea, ASL VC, Vercelli, Italy

29 Gastroenterology Unit, ASL TO3, Rivoli, Torino, Italy

30 Gastroenterology and Digestive Endoscopy Unit, ASST Bergamo Est, Bergamo, Italy

31 Gastroenterology and Endoscopy Unit, Fondazione IRCCS Ca' Granda Ospedale Maggiore Policlinico, Università degli Studi di Milano, Milan, Italy 
submitted 7.11.2020

accepted after revision 13.1.2021

\section{Bibliography \\ Endosc Int Open 2021; 09: E629-E634 \\ DOI 10.1055/a-1380-3419 \\ ISSN 2364-3722 \\ (c) 2021. The Author(s).}

This is an open access article published by Thieme under the terms of the Creative Commons Attribution-NonDerivative-NonCommercial License, permitting copying and reproduction so long as the original work is given appropriate credit. Contents may not be used for commercial purposes, or adapted, remixed, transformed or built upon. (https://creativecommons.org/licenses/by-nc-nd/4.0/)

Georg Thieme Verlag KG, Rüdigerstraße 14,

70469 Stuttgart, Germany

Corresponding author

Giulio Donato, MD, Gastroenterology Unit, AOU Maggiore della Carità, C.so Mazzini 18, 28100 Novara, Italy

Fax: +03213733345

giulio.donato@maggioreosp.novara.it

Supplementary material is available under https://doi.org/10.1055/a-1380-3419

\section{ABSTRACT}

Background and study aims COVID-19 has dramatically impacted endoscopy practice because upper endoscopy procedures can be aerosol-generating. Most elective proce- dures have been rescheduled. Endoscopic retrograde cholangiopancreatography (ERCP) is frequently performed in emergency or urgent settings in which rescheduling is not possible. We evaluated the impact of the COVID-19 pandemic on ERCP in Italy during the SARS-CoV-2 lockdown, in areas with high incidence of COVID-19.

Patients and methods We performed a retrospective survey of centers performing ERCP in high COVID-19 prevalence areas in Italy to collect information regarding clinical data from patients undergoing ERCP, staff, case-volume and organization of endoscopy units from March 8, 2020 to April 30, 2020.

Results We collected data from 31 centers and 804 patients. All centers adopted a triage and/or screening protocol for SARS-CoV-2 and performed follow-up of patients 2 weeks after the procedure. ERCP case-volume was reduced by $44.1 \%$ compared to the respective 2019 timeframe. Of the 804 patients undergoing ERCP, 22 (2.7\%) were positive for COVID-19. Adverse events occurred at a similar rate to previously published data. Of the patients, endoscopists, and nurses, $1.6 \%, 11.7 \%$, and $4.9 \%$, respectively, tested positive for SARS-CoV-2 at follow up. Only $38.7 \%$ of centers had access to a negative-pressure room for ERCP.

Conclusion The case-volume reduction for ERCP during lockdown was lower than for other gastrointestinal endoscopy procedures. No definitive conclusions can be drawn about the percentage of SARS-CoV-2-positive patients and healthcare workers observed after ERCP. Appropriate triage and screening of patients and adherence to society recommendations are paramount.

\section{Introduction}

In December 2019, a novel coronavirus, subsequently termed SARS-CoV-2, was detected in Wuhan, China, causing a severe respiratory disease named Coronavirus Disease (COVID-19). As a result of its ability to mainly spread through respiratory droplets, including those from asymptomatic individuals, SARSCoV-2 spread significantly worldwide, and on March 11, 2020, the World Health Organization (WHO) declared the infection a pandemic. Italy was the first European country to be severely involved with a high number of cases. Drastic measures had to be adopted to contain the spread of the virus, which was causing an overload of the national healthcare system. Crowd assemblage and events first were banned, then on March 8, the Italian government imposed a quarantine in the Northern regions with the highest incidence of COVID-19, rapidly followed by the extension of the lockdown to the whole country. A reduction in containment measures and a gradual resumption of activities, the so-called "phase two," were allowed beginning on May 4. By April 30, COVID-19 had infected more than 3 million people and caused 224,000 deaths worldwide, with more than 205,000 infected people and 28,000 deaths in Italy. At the time of the writing of this paper, those numbers had risen to more than 47 million people infected and 1.2 million deaths worldwide, with more than 750,000 infected people and 39,000 deaths in Italy [1].

The most common symptoms of COVID-19 include fever, cough, myalgia/fatigue, and dyspnea [2], but spread can occur during the incubation period ( 5 days on average, range $0-14$ ) and $80 \%$ of individuals are asymptomatic [3]. The case fatality rate was $5 \%$ in a Chinese meta-analysis [2]. Healthcare workers are up to three times more likely to contract COVID-19 than the general population and there were more than 20,000 such infections in Italy by April 30 [1]. These data clearly confirm that patients undergoing endoscopy might be asymptomatic carriers and preventive measures have to be taken into account to avoid human-to-human spread of the virus.

Recently, in addition to standard precautions, several strategies have been suggested to reduce the risk of SARS-CoV-2 transmission to patients and to endoscopy staff during gastrointestinal endoscopy (Box 1) [4-7].

All endoscopic procedures should be considered aerosolgenerating, owing to the possibility of coughing and retching during upper endoscopy, as well as the introduction of microdroplets through the instrument channels or leakage of valves [5]. After the COVID-19 outbreak, competent authorities im- 
BOX 1

Main recommendations for endoscopy in the COVID-19 era [5]

- Following general measures of physical distancing and adequate hand hygiene

- Postponing elective, non-urgent procedures

- Reducing endoscopy room staff to limit virus spread and conserve PPE and other resources

- Clear communication across the entire endoscopy team and training on endoscopy unit COVID protocols

- N95/FFP2/FFP3 respirators and a full set of PPE (gown, gloves, hair cover and goggles or face-shield) for the endoscopy staff

- Respect for the correct sequence of wearing and taking off PPE ("donning" and "doffing")

- Stratifying patients (triage/screening) before the procedure

- Surgical masking for all patients in endoscopy

- Following reprocessing protocols for endoscopes strictly

- Contacting patients 1 to 2 weeks after the procedure to check for symptoms

posed a drastic reduction in non-urgent activities performed in Italian hospitals to limit the spread of SARS-CoV-2 and to re-allocate healthcare professionals to other tasks. Elective and follow-up endoscopic procedures in Italy, therefore, were suspended, causing a $>60 \%$ reduction in total endoscopy activity, with peaks of $>90 \%$ reduction [8]. Endoscopic retrograde cholangiopancreatography (ERCP) is a challenging, predominantly therapeutic endoscopic-radiologic procedure, which is effective in relieving obstructive jaundice and also managing some iatrogenic surgical biliary complications. Despite the COVID-19 pandemic, ERCP is still requested for patients with ongoing biliary obstruction or infection, as rescheduling is often impossible in time-sensitive settings.

\section{Patients and methods}

The present study was a multicenter, retrospective, observational survey of Northern and Central Italy centers performing ERCP in high COVID-19 incidence and prevalence areas. Participating centers were contacted via email and invited to answer an electronic survey questionnaire (Supplementary material).

The participating centers were asked about their ERCP casevolume from March 8, 2020 to April 30, 2020 and in the same period during 2019 as a reference. Questions included organization (number of endoscopists, nurses and trainees, triage/ screening measures, sedation practice, reprocessing), facility (personal protective equipment [PPE], availability of a negative pressure endoscopic/radiological room) and clinical aspects (indications for ERCP, prevalence of COVID-19 patients, followup data).
Our aim was to evaluate the impact of the COVID-19 pandemic on ERCP procedure management in Italy.

\section{Results}

\section{Center characteristics}

From March 8 to April 30, 2020, 31 centers, all located in Northern and Central Italy, participated in this retrospective observational survey. The survey centers were located as follows: 25 $(80.6 \%)$ in the North and six (19.4\%) in the Center of Italy. The majority of centers were non-academic (28/31). Only two of 31 centers had a case-volume $<100$ ERCPs/year, while 21 of 31 centers performed > 250 ERCPs in 2019 (range 250-1109).

A negative pressure endoscopy room was available in five of 31 centers (16.1\%). Nine of 31 centers (26.0\%) performed ERCPs on SARS-CoV-2-positive patients in a separate room outside of the endoscopy department, seven of which had negative pressure.

\section{Population}

Data were retrospectively collected from a total of 804 patients (420 males, 384 females); 564 of 804 had an intact papilla. Of the 804 patients, 598 (74.4\%) were older than 60 years. Only 22 of 804 procedures $(2.7 \%)$ were performed in SARS-CoV-2positive patients. The number of procedures performed by the participating centers in the same period in 2019 was 1439.

The most frequent indication for ERCP was malignant biliary obstruction from distal tumors $(213 / 804,26.5 \%)$, followed by common bile duct (CBD) stones (190/804, 23.6\%) and cholangitis $(152 / 804,18.9 \%)$. See $>$ Table 1 for all indications.

\section{Medical/nurse staffing}

There were 69 experienced endoscopists performing ERCP (range 1-4 per center); there were 34 trainees (range $0-2$ per center). Training in ERCP was suspended in six of the 27 centers with an ongoing training program. Trainees continued their usual ERCP training in 11 of 27 centers, while it was reduced in 10 of 27 centers. The main reasons for discontinuation or reduction in ERCP training were reassignment of trainees to COVID wards and limitations in availability of PPE. There were 183 ERCP nurses (range 2-16 per center). During the lockdown period, staff members in all centers were also involved in other endoscopic or clinical activities, sometimes including COVID ward support.

\section{Triage/screening}

All centers implemented a specific questionnaire triage (travel to high-risk areas or contact with COVID-19 patients; presence of COVID-19 symptoms) and/or carried out a screening protocol prior to ERCP to check and stratify the risk of COVID-19 in patients. A nasopharyngeal swab was used as a screening test in all centers except one (30/31, 96.8\%). Twenty-five of 30 centers $(83.3 \%)$ performed a swab on all patients, while five of 30 (16.7\%) carried out the screening only in the presence of symptoms or after a positive triage questionnaire. Twenty-three of 30 centers $(76.7 \%)$ performed further screening with a chest computed tomography scan for positive swab and/or sympto- 


\begin{tabular}{|c|c|}
\hline & Total $(n=804)$ \\
\hline Indication for ERCP & n (\%) \\
\hline Stenting for distal tumors & $213(26.5)$ \\
\hline CBD stones & $190(23.6)$ \\
\hline Cholangitis & $152(18.9)$ \\
\hline Biliary stent replacement & $62(7.7)$ \\
\hline Acute pancreatitis & $54(6.7)$ \\
\hline Perihilar cholangiocarcinoma & $28(3.5)$ \\
\hline latrogenic biliary leak/stricture & $23(2.9)$ \\
\hline Ampullary neoplasia & $22(2.7)$ \\
\hline Papillary stenosis of unknown origin & $13(1.6)$ \\
\hline Biliary stenosis in chronic pancreatitis & $9(1.1)$ \\
\hline Post-OLT biliary stenosis & $7(0.9)$ \\
\hline Recurrent pancreatitis in pancreas divisum & $6(0.7)$ \\
\hline Pancreatic stent replacement & $5(0.6)$ \\
\hline Main pancreatic duct stenosis or stone & $5(0.6)$ \\
\hline Hemobilia/papillary bleeding & $3(0.4)$ \\
\hline $\begin{array}{l}\text { Other (Mirizzi's syndrome, main pancreatic } \\
\text { duct disruption, Recurrent pancreatitis, PSC, } \\
\text { extrinsic neoplastic compression of CBD, peri- } \\
\text { papillary GIST) }\end{array}$ & $12(1.5)$ \\
\hline \multicolumn{2}{|c|}{$\begin{array}{l}\text { ERCP - endoscopic retrograde cholangiopancreatography; CBD - common } \\
\text { bile duct; OLT - orthotopic liver transplantation; PSC - primary sclerosing } \\
\text { cholangitis; GIST - gastrointestinal stromal tumor. }\end{array}$} \\
\hline
\end{tabular}

matic patients with a negative swab using variable local protocol policies.

\section{Personal protective equipment (PPE)}

FFP2/3 respirators were used by the endoscopy staff on all patients in 30 of 31 centers (96.8\%); one of 31 reserved their use for SARS-CoV-2-positive patients only. Operators wore waterresistant gowns for all procedures in $87.1 \%$ of centers and only for SARS-CoV-2-positive procedures in the remaining $12.9 \%$. A face shield was used in all except one center (96.8\%). A surgical hair cap was used in $93.5 \%$ of centers. Operators wore two pairs of gloves in $87.1 \%$ of centers.

\section{Sedation practice}

Deep sedation was the most common sedation modality (13/ $31,41.9 \%)$. Five of 31 centers (16.1\%) shifted their sedation practice to general anesthesia for COVID-19 patients.

\section{Reprocessing}

The pandemic triggered an optimization of adherence to endoscope reprocessing protocols in seven of 31 centers (22.6\%).

\section{Follow-up data}

All centers performed a phone call follow-up of patients 2 weeks after each procedure to record adverse events (AEs) or COVID-19 symptom development. At the 2-week follow-up time-point, 13 previously SARS-CoV-2-negative patients tested positive (13/804, 1.6\%), of whom five were asymptomatic.

As regards the endoscopy staff, the cumulative incidence of SARS-CoV-2 during the lockdown timeframe was $11.7 \%$ among endoscopists (12/103, of whom 1 was a trainee) and $4.9 \%$ among nurses (9/183). Two of 12 SARS-CoV-2-positive endoscopists and three of 10 SARS-CoV-2 positive nurses were performing COVID wards shifts.

\section{ERCP adverse events}

Twenty-three of 804 patients (2.9\%) developed post-ERCP acute pancreatitis (PEP) and bleeding occurred in 16 of 804 (2.0\%); however, none of these AEs was severe. An infectious $\mathrm{AE}$ (cholangitis/cholecystitis) occurred in 14 of 804 patients $(1.7 \%)$, of which four were severe. All four reported cases of perforation $(0.5 \%)$ required surgical management. Three of $804(0.4 \%)$ died as a consequence of COVID-19; there were no ERCP-related deaths.

\section{Discussion}

ERCP often is performed for acute biliary obstruction in emergent settings such as acute cholangitis or urgent, time-sensitive settings such as acute biliary pancreatitis, obstructive jaundice in malignant stenosis, symptomatic CBD gallstones or biliary leaks after surgery. Rescheduling of ERCP is not possible in these scenarios. Moreover, ERCP is more time-consuming than other gastrointestinal endoscopy procedures and it is often scheduled in tandem with endoscopic ultrasound (EUS), another time-consuming procedure. Last but not least, ERCP patients might require hospitalization. In the event of a SARSCoV-2-positive patient, all these factors add up to a potential increase in exposure for both the patients and healthcare workers linked to ERCP.

The endoscopy centers participating in our survey showed a medium to high annual case-volume for ERCP. There was a significant reduction $(44.1 \%)$ in the number of procedures from March 8 to April 302020 compared to the same period in 2019. This might be explained by a rescheduling of some of the elective indications for ERCP (stent replacement, treatment of recurrent pancreatitis, etc.) but there also might have been a reduction in medical attendance by patients with abdominal symptoms, as a consequence of fear of contracting the virus in hospitals. The reduction in ERCPs, however, was less than for other endoscopy procedures, which even reached $90 \%$ in some areas [8].

Although triage/screening was not standardized and there was some variability among centers, each center, at a minimum, implemented a screening protocol prior to ERCP to provide patient risk stratification to prevent the spread of SARSCoV-2 and use proper measures when scheduling the procedure. All centers also performed follow-up phone calls, which 
are a very useful tool, albeit imperfect, for keeping track of patient symptoms and AEs. A small percentage of centers also optimized adherence to endoscope reprocessing protocols, which is a key element in containing biological risks. Endoscope transmission of any kind of virus is reasonably rare if we thoroughly follow current guidelines in reprocessing. PPE use was generally adherent to society and expert recommendations. Although performing endoscopy procedures in a negative-pressure room was recommended by gastroenterology societies guidelines both before [9] and after [5] the pandemic outbreak, only $38.7 \%$ of centers had access to negative-pressure rooms for SARS-CoV-2-positive patients. There is an urgent need to update facilities to this standard protection against airborne pathogens.

No definitive conclusions can be drawn about the observed relatively high percentage of healthcare workers who tested positive for SARS-CoV-2 during the 2-week follow up, because tracking movements and contacts of patient and endoscopy staff was not implemented and the endoscopy room is just one place where the ERCP patient and staff spent time. Moreover, some operators were also involved in COVID wards shifts and they all came from areas with a high prevalence of COVID19. On the contrary, the small percentage of patients who tested positive at follow-up despite coming from the same high prevalence areas and being susceptible to the same observations made for operators, might be a clue that triage/screening procedures and PPE use are adequate prevention tools.

As this survey showed, the ERCP volume load was not reduced as much as other endoscopic activities, because of the aforementioned pivotal role of ERCP in some emergent and urgent settings. We, therefore, believe that ERCP personnel should be "protected" by healthcare authorities and should not be exposed to any increased risk of infection during a pandemic, if we take into account the paucity of ERCP operators in a country. We recognize that establishing an official priority among healthcare personnel is a tough task, as it should be based on evidence, shared by the majority or imposed, and it might be unpopular to some. However, local healthcare authorities might identify life-saving procedures performed in each center and involved healthcare operators to consider and have remain in place when workers are reallocated to pandemic-focused activities.

The rate of AEs was comparable to previously published data. PEP occurred at a very low rate $(2.9 \%)$ and no cases were severe, which met the European Society of Gastrointestinal Endoscopy (ESGE) performance measure target of <10\% [10]. The bleeding rate $(2.0 \%)$ was a little higher than the ASGE post-procedural indicator of $<1 \%$ [11], but comparable to all of the most recent reports (up to $3.6 \%$ ) $[12,13]$, and there were no severe cases. The rate of perforation was $0.5 \%$, again higher than the ASGE indicator of $<0.2 \%$ but comparable to the $0.6 \%$ rate in an Austrian survey [12]. No ERCP-related deaths were reported.

This survey study had some limitations. The first was the retrospective design. The second was the limited number of centers/cases: only a very small percentage of patients (2.7\%) were SARS-CoV-2-positive. Third, because of the short follow- up period and the lack of a standardized reassessment of SARS-CoV-2 status, data on the virus spread might have been underestimated; however, thorough epidemiological tracking of cases is very hard to implement, especially when the prevalence of the virus is high in the population, as in our reality.

\section{Conclusions}

In conclusion, to the best of our knowledge, this was the first multicenter survey in Europe to collect organizational, clinical, and outcome data on ERCP in the COVID-19 era in high prevalence areas. The impact of COVID-19 on gastrointestinal endoscopy procedure volumes was significant but not extreme for ERCP. This procedure can be life-saving in emergent settings and it cannot be rescheduled for most of the other time-sensitive indications. For these same reasons, healthcare authorities should take the importance of ERCP operators into account when reallocating staff resources during pandemics. In an effort to reduce the spread of SARS-CoV-2 before, during, and after endoscopy procedures, accurate triage, screening, and actuation of society recommendations are our only weapons, at present. Strict adherence to these measures may also prevent the spread of SARS-CoV-2 during the subsequent period of resumption of normal activities.

\section{Competing interests}

The authors declare that they have no conflict of interest.

\section{References}

[1] Covid-19: Situazione in Italia. Available from: http://www.salute.gov. it/portale/nuovocoronavirus/homeNuovoCoronavirus.jsp?lingua=english

[2] Li LQ, Huang T, Wang YQ et al. COVID-19 patients' clinical characteristics, discharge rate, and fatality rate of meta-analysis. J Med Virol 2020: doi: $10.1002 / j m v .25757$

[3] Wang Y, Wang Y, Chen Y et al. Unique epidemiological and clinical features of the emerging 2019 novel coronavirus pneumonia (COVID19) implicate special control measures. J Med Virol 2020: doi: $10.1002 / j m v .25748$

[4] Gralnek IM, Hassan C, Beilenhoff U et al. ESGE and ESGENA Position Statement on Gastrointestinal Endoscopy and the COVID-19 Pandemic. Endoscopy 2020; 52: 483-490

[5] Joint Gastroenterology Society Message: COVID-19 Use of Personal Protective Equipment in GI Endoscopy (AASLD, ACG, AGA. ASGE). 2020: https://www.asge.org/home/joint-gi-society-message-covid19

[6] Soetikno R, Teoh AYB, Kaltenbach T et al. Considerations in performing endoscopy during the COVID-19 pandemic. Gastrointest Endosc 2020: doi:10.1016/j.gie.2020.03.3758

[7] Repici A, Maselli R, Colombo M et al. Coronavirus (COVID-19) outbreak: what the department of endoscopy should know. Gastrointest Endosc 2020: doi:10.1016/j.gie.2020.03.019

[8] Maida M, Sferrazza S, Savarino E et al. Impact of the COVID-19 Pandemic on Gastroenterology Divisions in Italy: A National Survey. Dig Liver Dis 2020: doi:10.1016/j.dld.2020.05.017 
[9] Calderwood AH, Day LW. ASGE Quality Assurance in Endoscopy Committee. et al. ASGE guideline for infection control during Gl endoscopy. Gastrointest Endosc 2018; 87: 1167-1179

[10] Domagk D, Oppong KW, Aabakken L et al. Performance measures for endoscopic retrograde cholangiopancreatography and endoscopic ultrasound: A European Society of Gastrointestinal Endoscopy (ESGE) Quality Improvement Initiative. United European Gastroenterol J 2018; 6: 1448-1460
[11] Adler DG, Lieb JG 2nd, Cohen J et al. Quality indicators for ERCP. Gastrointest Endosc 2015; 81: 54-66

[12] Kapral C, Mühlberger A, Wewalka F et al. Quality assessment of endoscopic retrograde cholangiopancreatography: results of a running nationwide Austrian benchmarking project after 5 years of implementation. Eur J Gastroenterol Hepatol 2012; 24: 1447-1454

[13] Mariani A, Segato S, Anderloni A et al. Prospective evaluation of ERCP performance in an Italian regional database study. Dig Liver Dis 2019; 51: $978-984$ 